\title{
Elucidating the 3d Electronic Configuration in Manganese Phthalocyanine
}

Iulia Emilia Brumboiu ${ }^{1}$, Roberta Totani ${ }^{2}$, Monica de Simone ${ }^{3}$, Marcello Coreno ${ }^{4}$, Cesare Grazioli ${ }^{3,5}$, Luca Lozzi ${ }^{2}$, Heike C. Herper ${ }^{1}$, Biplab Sanyal ${ }^{1}$, Olle Eriksson ${ }^{1}$, Carla Puglia ${ }^{1}$, and Barbara Brena ${ }^{1, *}$

${ }^{1}$ Department of Physics and Astronomy, Uppsala University, Box-516, SE-75120 Uppsala, Sweden

${ }^{2}$ Department of Physical and Chemical Sciences, University of L'Aquila, Via Vetoio, 67100 L'Aquila, Italy

${ }^{3}$ CNR-IOM, Laboratorio TASC, Sincrotrone Trieste, S.S. $14 \mathrm{Km} \mathrm{163.5,}$ Basovizza, I-34149 Trieste, Italy

${ }^{4}$ CNR-IMIP, Montelibretti c/o Sincrotrone Trieste, S.S. 14 Km 163.5, Basovizza, I-34149 Trieste, Italy

${ }^{5}$ Department of Chemical and Pharmaceutical Sciences, University of Trieste, Italy

*Corresponding Author: barbara.brena@physics.uu.se.

\begin{abstract}
To shed light on the metal $3 \mathrm{~d}$ electronic structure of manganese phthalocyanine, so far controversial, we performed photoelectron measurements both in the gas phase and as thin film. With the purpose of explaining the experimental results, three different electronic configurations close in energy to one another were studied by means of density functional theory. The comparison between the calculated valence band density of states and the measured spectra revealed that in the gas phase the molecules exhibit a mixed electronic configuration, while in the thin film, manganese phthalocyanine finds itself in the theoretically computed ground state, namely, the $b^{1} e^{3} a^{1} b^{0}$ electronic configuration.
\end{abstract}





\section{INTRODUCTION}

Organic molecules with a $3 \mathrm{~d}$ transition-metal center have become significant owing to the fact that they play a major role in a wide variety of emerging fields such as organic electronics ${ }^{1}$ and spintronics. $^{2}$ In particular, manganese phthalocyanine $(\mathrm{MnPc})$ is an organic semiconductor and molecular magnet able to act as a spin-filter. ${ }^{3}$ Moreover, its spin can be manipulated in a controlled manner, ${ }^{4-6,8}$ giving rise to its possible use in molecular spintronics ${ }^{7}$ and quantum computing applications..$^{9-11}$ In fact, it has been recently shown that the spin polarization of a single $\mathrm{MnPc}$ molecule may be controlled either by the chemical coordination of ligands to the central metal ${ }^{4,8}$ or according to the interplay between Kondo screening and superconducting pair formation. ${ }^{6,12}$ In this context, not only would a precise knowledge of the exact metal 3d electronic configuration of MnPc shed light on the previously mentioned effect but it would also prove to be essential in view of spintronics applications by opening the possibility of controlling the orientation of the magnetic anisotropy of the molecule. In fact, it has been recently proven that the addition or removal of ligands could be used to modulate the direction of the spontaneous magnetization. ${ }^{13}$ Because the highest occupied $3 \mathrm{~d}$ orbital and, respectively, the lowest unoccupied bring, in general, the largest contribution to the anisotropy, understanding the manner in which the metal states are populated may prove to be essential in both clarifying the origins of the molecular magnetic anisotropy as well as in reversibly controlling its orientation (from uniaxial to in plane and vice versa).

As opposed to the majority of transition metal phthalocyanines (TMPCs), for $\mathrm{MnPc}$, the high structural symmetry is broken in a Jahn-Teller (JT) manner. ${ }^{14}$ The molecule exhibits $\mathrm{D}_{2 \mathrm{~h}}$ symmetry because an odd number of electrons populates the doubly degenerate $\mathrm{e}_{\mathrm{g}}$ molecular state, making $\mathrm{MnPc}$ an interesting model for analyzing the correlation between electronic structure and molecular geometry in view of the JT effect. 
According to ligand-field theory, a transition metal placed into a $\mathrm{D}_{4 \mathrm{~h}}$ symmetric environment will exhibit a splitting of its $3 \mathrm{~d}$ levels, as depicted in the inset of Figure 1. Controversy exists regarding the manner in which these states are populated to give rise to the intermediate spin $(\mathrm{S}=3 / 2)$ of $\mathrm{MnPc}$. There are three possibilities of metal $3 \mathrm{~d}$ orbital occupation that have been discussed, which all leave the $d_{x^{2}-y^{2}}$ level unoccupied: the $b^{1} e^{3} a^{1} b^{0}$ electronic configuration (denoted $S_{A}$ here), $b^{2} e^{2} a^{1} b^{0}$ (denoted Sв ), and $b^{1} e^{2} a^{2} b^{0}$ (denoted Sc ). Several experimental studies performed at different temperatures on either $\mathrm{MnPc}$ single crystal or $\mathrm{MnPc}$ deposited on noble-metal substrates report not only different electronic configurations of the metal but also different symmetries of the highest occupied molecular orbital (HOMO). More specifically, two studies, one using X-ray absorption spectroscopy (XAS) ${ }^{17}$ and the other using both magnetic circular dichroism (MCD) and absorption spectroscopy, ${ }^{18}$ report the $S\left(b^{1} e^{3} a^{1} b^{0}\right)$ electronic configuration as the ground state, while a third one performed by XAS and X-ray $\mathrm{MCD}^{19}$ confirms this configuration but reveals a different HOMO, namely, the $\mathrm{a}_{1 \mathrm{~g}}$. An L-edge XAS study ${ }^{23}$ reports two electronic configurations in competition $\left(\mathrm{S}_{\mathrm{A}}\right.$ and $\left.\mathrm{S}_{\mathrm{B}}\right)$, whereas a different magnetic experiment ${ }^{22}$ reveals only $\mathrm{S}_{\mathrm{B}}\left(\mathrm{b}^{2} \mathrm{e}^{2} \mathrm{a}^{1} \mathrm{~b}^{0}\right)$ as the ground state.

Similar controversy exists in density functional theory (DFT) studies performed using different exchange and correlation functionals, basis sets, and atomic orbital types. A $\mathrm{b}^{1} \mathrm{e}^{3} \mathrm{a}^{1}$ ground state is reported by Liao et al. as well as by Wang et al. ${ }^{21}$ Marom and Kronik, ${ }^{14}$ by enforcing symmetry, reveal the different character of the Mn highest occupied d level. An $\mathrm{e}_{\mathrm{g}} \mathrm{HOMO}$ is reported when the symmetry is broken, while by conserving symmetry they obtain either a $b_{2 g}$ or an $a_{1 g} \mathrm{HOMO}$, the two structures being close in energy and competing. In addition, a joint theoretical (DFT) and experimental (valence band photoemission at $21.2 \mathrm{eV}$ photon energy) study ${ }^{15}$ reports an $\mathrm{e}_{\mathrm{g}}$ symmetry for the HOMO level.

In the present study, the $\mathrm{S}_{\mathrm{A}}, \mathrm{S}_{\mathrm{B}}$, and $\mathrm{S}_{\mathrm{C}}$ electronic configurations were analyzed by 
means of DFT with the B3LYP hybrid functional to clarify the controversy. The computed valence band spectra were compared with photoelectron spectroscopy (PES) measurements performed at different photon energies in both gas phase and thin film. In this way, exploiting the variation of the atomic orbital cross sections for different photon energies, we could obtain a detailed knowledge of the elemental composition of the electronic states in the valence band.

\section{EXPERIMENTAL SECTION}

The PES measurements on the gas-phase sample were performed at the GAsPHase (GAPH) beamline of the Elettra synchrotron in Trieste, ${ }^{24}$ using a VG analyzer mounted at $54.7^{\circ}$ with respect to the electric vector of the light. ${ }^{25}$ Manganese(II) phthalocyanine (MnPc) (powder, purity 95\%) was purchased from Sigma Aldrich. The molecules were further purified in situ before the experiment to reduce traces of contaminants. ${ }^{26}$ The sample was sublimated using a custom built resistively heated furnace. The MnPc molecules were evaporated at $314 \pm 1$ ${ }^{\circ} \mathrm{C}$, avoiding dissociation observed at higher temperatures. Calibration of the binding energy of the outer-valence spectra was done by He (vertical ionization energy, $\mathrm{VIE}=24.59 \mathrm{eV})$ and by traces of vaporized water $(\mathrm{VIE}=12.62 \mathrm{eV})^{27}$ found in the system. During the whole experiment, the pressure at the ionization region remained constant (ca. $8 \times 10 \mathrm{mbar}$ ). $\mathrm{PE}$ spectra have been normalized to the photon flux and to the analyzer transmission function. The thin-film experimental measurements were performed for $\mathrm{MnPc}$ deposited on $\mathrm{Au} / \mathrm{mica}$ substrate purchased from George Albert Physikal Vapor Disposition. Before the $\mathrm{MnPc}$ deposition, the surface was cleaned by several cleaning cycles $\left(\mathrm{Ar}^{+}\right.$ sputtering, followed by annealing at $\left.450{ }^{\circ} \mathrm{C}\right)$, resulting in a reconstructed $\mathrm{Au}(111)$ surface. The MnPc molecules, the same used in gas-phase measurements, were 
degassed and thermally evaporated from a quartz crucible under ultrahigh vacuum conditions (base pressure approximately $10^{-10} \mathrm{mbar}$ ). The evaporation temperature used did not exceed $315{ }^{\circ} \mathrm{C}$ to avoid the molecule decomposition. A monochromatic X-ray source ( $\mathrm{Al} \mathrm{K \alpha}, 1486.7 \mathrm{eV}$ ), a He discharge lamp (21.2 eV), and a hemispherical analyzer were used to perform XPS and UPS measurements. The binding energy scale was calibrated with the Fermi level of the gold substrate.

\section{COMPUTATIONAL METHODS}

From the theoretical point of view, single-molecule DFT calculations were performed using the Gaussian 09 program. ${ }^{28}$ A full relaxation of all atoms was allowed. The hybrid B3LYP exchange-correlation functional ${ }^{29}$ was used along with the $6-31 \mathrm{G}(\mathrm{d}, \mathrm{p})$ basis set (valence double- $\zeta$ plus polarization) ${ }^{30}$ for carbon, nitrogen, and hydrogen atoms, while for $\mathrm{Mn}$, the cc-pVTZ (valence triple- $\zeta$ plus polarization) $)^{31}$ was employed. Starting from $\mathrm{D}_{4 \mathrm{~h}}$ symmetry, the system relaxes to $\mathrm{D}_{2 \mathrm{~h}}$ symmetry in the case of the $\mathrm{S}_{\mathrm{A}}$ electronic configuration. The other two electronic structures are obtained by first depopulating the highest occupied molecular orbital (HOMO) and then occupying a spin-down unoccupied level, followed by a structure optimization. Both of the resulting relaxed geometries are very close to the $\mathrm{D}_{4 \mathrm{~h}}$ point group (Figure $2 \mathrm{c}$ ). The total and partial density of states (DOS) for the three different electronic configurations was determined using the $\mathrm{c}^{2}$ population analysis (SCPA) method, ${ }^{32}$ as performed in a previous study. ${ }^{33}$ The partial density of states (PDOS) was used to simulate the photoelectron valence band spectra at different photon energies. For this purpose, the PDOS for each atomic orbital was multiplied by its corresponding photoionization cross section at the specific photon energy ${ }^{34-37}$ and a total DOS (TDOS) for this specific energy was determined. To facilitate the comparison with experiments, the theoretical bar 
graphs were broadened using Gaussian functions. For Figure 3, the calculated DOS were broadened using a $0.5 \mathrm{eV}$ initial full width at half-maximum (fwhm), which was linearly increased to $2.0 \mathrm{eV}$ in the range -5.0 to $-15.0 \mathrm{eV}$ and then kept constant. The $21.2 \mathrm{eV}$ photon energy simulated spectra were shifted by $3.33 \mathrm{eV}$ and the $1486.7 \mathrm{eV}$ results were shifted by $3.8 \mathrm{eV}$ toward lower binding energies to align the calculated spectra to the experimental first peak. In Figure 4, a Gaussian broadening was performed starting with a $0.3 \mathrm{eV}$ initial fwhm, which was linearly increased to $2.0 \mathrm{eV}$ in the range of -6.5 to $-10.0 \mathrm{eV}$ and then kept constant. The spectra were normalized to a $C 2 p$ peak centered at $-12.1 \mathrm{ev}$. The mixed spectra were shifted with $1.37 \mathrm{eV}$ to higher binding energies. Similarly, $\mathrm{S}_{\mathrm{A}}$ was shifted by $1.37 \mathrm{eV}, \mathrm{S}_{\mathrm{B}}$ by $1.31 \mathrm{eV}$, and $\mathrm{S}_{\mathrm{C}}$ by $1.6 \mathrm{eV}$, all of them toward higher binding energies to match the first experimental peak.

\section{RESULTS AND DISCUSSION}

According to the computed total energy, the $\mathrm{S}_{\mathrm{A}}$ electronic configuration corresponds to the ground state of the intermediate spin MnPc. $\mathrm{S}_{\mathrm{B}}$ and $\mathrm{S}_{\mathrm{C}}$ are slightly higher in energy, the first one by $0.11 \mathrm{eV}$ and the second by $0.23 \mathrm{eV}$. Structurally, the main differences between the three structures concern the $\mathrm{Mn}-\mathrm{N}$ as well as the $\mathrm{C}-\mathrm{N}$ bond lengths. The relevant bonds are shown in Figure 1, where the different types of $\mathrm{N}$ atoms are labeled as $\mathrm{N}_{\mathrm{i}}$ and $\mathrm{N}_{\mathrm{m}}$, and the different $\mathrm{C}$ atoms are labeled $\mathrm{C}_{\alpha}$ and $\mathrm{C}_{\beta}$. The largest change in bond length for the different electronic configurations is the $\mathrm{Mn}-\mathrm{N}_{\mathrm{i}}$ bond length (denoted "a" in Figure 1), which varies between $1.957 \AA$ for $\mathrm{S}_{\mathrm{A}}$, to $1.971 \AA$ for $\mathrm{S}_{\mathrm{B}}$, and $1.967 \AA$ for $\mathrm{S}_{\mathrm{C}}$. Other differences, not as remarkable, involve the pyrrole $\mathrm{C}_{\alpha}$ atom ("b", "c", and "d" bond lengths in Figure 1). More precisely, the $\mathrm{C}_{\alpha}-\mathrm{N}_{\mathrm{i}}$ bond length is $1.384 \AA$ in the case of $\mathrm{S}_{\mathrm{A}}$ decreasing to $1.378 \AA$ for the other two electronic configurations. The 
$\mathrm{C}_{\alpha}-\mathrm{N}_{\mathrm{m}}$ bond has a length of $1.324 \AA$ in the ground state, changing to $1.328 \AA$ for $\mathrm{S}_{\mathrm{B}}$ and to $1.326 \AA$ for $\mathrm{S}_{\mathrm{C}}$. Finally, the $\mathrm{C}_{\alpha}-\mathrm{C}_{\beta}$ bond length varies between $1.453 \AA$ $\left(\mathrm{S}_{\mathrm{A}}\right), 1.457 \AA\left(\mathrm{S}_{\mathrm{B}}\right)$, and $1.456 \AA\left(\mathrm{S}_{\mathrm{C}}\right)$.

A comparison between the Mn 3d PDOS of the three structures in the HOMO-LUMO region shows that the $\mathrm{d}_{\mathrm{x}^{2}-\mathrm{y}^{2}}$ orbital is high in energy and remains unoccupied for both spin channels (Figure 2a). Differences between $S_{A}, S_{B}$, and $S_{C}$ are related to the character of the HOMO, to its position, and to the HOMO-LUMO gap (defined as the energy difference between the lowest unoccupied level in the spin down channel and the highest occupied level of the same spin) (Table 1). The $\mathrm{S}_{\mathrm{A}} \mathrm{HOMO}$ is a partially filled doubly degenerate $\mathrm{e}_{\mathrm{g}}$ orbital $\left(\mathrm{d}_{\mathrm{xz}}, \mathrm{d}_{\mathrm{yz}}\right)$ with an eigenvalue of $-4.46 \mathrm{eV}$. The calculated HOMO- LUMO gap is $1.35 \mathrm{eV}$. For the $\mathrm{S}_{\mathrm{B}}$ electronic configuration, the HOMO, exhibiting $\mathrm{b}_{2 \mathrm{~g}}$ character, is centered at $-4.59 \mathrm{eV}$ and the HOMO-LUMO gap becomes $1.65 \mathrm{eV}$. Finally, the $\mathrm{S}_{\mathrm{C}} \mathrm{HOMO}$ has $\mathrm{a}_{1 \mathrm{~g}}$ symmetry and is higher in energy than the other two $(-4.31 \mathrm{eV})$ with the smallest HOMO-LUMO gap $(1.32 \mathrm{eV})$.

Figure $2 \mathrm{~b}$ shows a comparison between the computed TDOS of the three electronic structures. The origin of the peaks is analyzed by comparing the TDOS to the Mn 3d and C 2p partial densities of states. The N 2p PDOS is presented in the Supporting Information. The main features of the spectra are denoted A-G, ${ }^{38}$ while the peaks in each region are designated with numbers. It is very important to note that $S_{A}, S_{B}$, and $S_{C}$ differ strongly in the Mn 3d states, a fact that generates DOS curves with different profiles for the three electronic structures. The main differences between them arise in the low binding energy range of the spectra (features $\mathrm{A}-\mathrm{C}$ ). More specifically, feature $\mathrm{A}$, situated in the HOMO region and consisting of two peaks $\left(A_{1}\right.$, of $M n 3 d$ character, and $A_{2}, C 2 p$ related), exhibits different $A_{1}-A_{2}$ splitting for each electronic structure. $S_{C}$ displays the largest splitting $(0.65 \mathrm{eV})$, as the HOMO is shifted to lower binding energies, while the A peaks of $S_{B}$ are very close together $(0.3 \mathrm{eV})$ because the HOMO is slightly shifted 
to higher binding energies. The ground-state electronic structure $\left(\mathrm{S}_{\mathrm{A}}\right)$ presents an intermediate splitting of $0.47 \mathrm{eV}$. Equally important differences arise in the B region. First of all, the $B_{1}$ peak, exhibiting $\mathrm{Mn} 3 \mathrm{~d}$ character in all three cases, is hardly visible in the TDOS of the ground-state structure. Instead, in the $\mathrm{S}_{B}$ spectrum, it is easily distinguishable and it becomes a relatively intense double peak in the $\mathrm{S}_{\mathrm{C}}$ electronic configuration. Second, in the upper binding energy region of feature B, the Mn 3d bands are present at different positions for each of the three electronic configurations. More precisely, in the $\mathrm{S}_{\mathrm{A}}$ case, the metal states contribute to peaks $B_{4}$ and $B_{5}$, while in $S_{B}$ case, the slightly less intense Mn bands are shifted to lower binding energies and contribute to peaks $B_{2}$ and $B_{3}$. In the $S_{C}$ TDOS, $\mathrm{B}_{3}$ has $\mathrm{Mn} 3 \mathrm{~d}$ character, similarly to peak $\mathrm{B}_{5}$, which is shifted toward region C. Finally, another important distinction between the three electronic configurations is the fact that the ground-state structure exhibits large Mn 3d character in the $\mathrm{C}$ feature, while $\mathrm{S}_{\mathrm{B}}$ and $\mathrm{S}_{\mathrm{C}}$ lack metal $3 \mathrm{~d}$ states in the same region. The previously mentioned differences are strongly relevant when comparing the computed spectra with PES measurements performed at different photon energies. Figure 3 displays a comparison between the calculated results and the thin-film experiments performed at $21.2 \mathrm{eV}$ (UPS) and $1486.7 \mathrm{eV}$ (XPS), respectively. At $21.2 \mathrm{eV}$ photon energy, all three calculated spectra compare well with the experiment, and they highly resemble one another. In fact, S, S , and S have very similar C 2p PDOS, and at this photon energy the excitations from the $\mathrm{C} 2 \mathrm{p}$ levels are much more probable than those from metal states.

An interesting conclusion comes when comparing the theory with the XPS (1486.7 $\mathrm{eV}$ ) experiment. In this case, the Mn $3 \mathrm{~d}$ states become dominant, and it is precisely in this feature that the three structures differ, as previously discussed. The shape and intensity ratios of the TDOS peaks of the $\mathrm{S}_{\mathrm{A}}$ structure compare well with the experiment, while the other two calculated spectra are noticeably different. Both 
$S_{B}$ and $S_{C}$ configurations overestimate peaks $B_{1}$ and $A_{1}$. Moreover, feature $C$ is strongly underestimated in both. This shows that in the thin films investigated here the MnPc displays the $b^{1} e^{3} a^{1} b^{0}\left(S_{A}\right)$ electronic configuration, in agreement with previous experimental studies. ${ }^{17,18}$ On the contrary, in the gas phase, a mixed electronic structure manifest itself, as will be discussed in the following.

The gas-phase PES results at different photon energies are compared with calculated spectra obtained by summing the density of states of two electronic configurations $\left(S_{A}\right.$ and $S_{B}$, Figure 4). The DOS of $S_{A}$ and $S_{B}$ configurations enter with equal weight in the total theoretical spectrum. The reason for considering the possible copresence of more than one electronic configuration in the same measurement is the fact that the features of the experimental results are not present in any of the individual calculated spectra of all configurations, $\mathrm{S}_{\mathrm{A}}, \mathrm{S}_{\mathrm{B}}$, or $\mathrm{S}_{\mathrm{C}}$. This is revealed in Figure 4c, displaying a comparison of the $100 \mathrm{eV}$ photon energy computed results to the gas-phase experiment performed at the same excitation energy. The $S_{A}$ spectrum lacks structure in the $B_{2}$ region, and peaks $B_{2}$ and $B_{1}$ do not match the position of the corresponding experimental bands. The $\mathrm{S}_{B}$ reproduces well the $B_{2}$ region, but the $B_{1}$ peak is much too intense and $A_{2}$ completely vanishes. As for the $S_{C}$ structure, it displays a largely overestimated $A_{1}$ band and a double $\mathrm{B}_{1}$ peak higher in intensity than the rest of the $\mathrm{B}$ region.

As opposed to the pure electronic configurations, the spectra obtained by summing the TDOS of two electronic configurations compare well with the experiment and reproduce the observed trends, namely, the intensity growth of the $A_{1}$ and $B_{1}$ peaks. A presence of a small percentage of the $S_{C}$ electronic configuration as well as of the metal-free phthalocyanine cannot be excluded, but for the current study, this percentage was not quantifiable; therefore, the $\mathrm{S}_{\mathrm{C}}$ structure and $\mathrm{H}_{2} \mathrm{Pc}$ were left out. This shows that in the gas-phase experiments the MnPc molecules find themselves in either $S_{A}$ or $S_{B}$ electronic configuration. During the gas-phase measurements, the molecules are heated to $300{ }^{\circ} \mathrm{C}$, having a thermal energy 
approaching our calculated energy difference between the $S_{A}$ and $S_{B}$ configurations. Hence Maxwell-Boltzmann statistics at this temperature would provide a mixture of molecules in configuration $\mathrm{S}_{\mathrm{A}}$ and $\mathrm{S}_{\mathrm{B}}$. It is not unlikely that the calculations overestimate by a small amount the energy difference between these two configurations, and, in this case, the thermal energy could provide a rather equal distribution of the two configurations.

\section{CONCLUSIONS}

To summarize, DFT calculations of three Mn 3d electronic configurations were performed, and the calculated valence band spectra were compared with PES measurements of the $\mathrm{MnPc}$ thin film and gas phase at different photon energies. The main result of the present study is that in the thin film, MnPc finds itself in the calculated electronic ground state $b^{1} e^{3} a^{1} b^{0}$, while in the gas phase it exhibits a mixed electronic configuration $\left(\mathrm{S}_{\mathrm{A}}\right.$ and $\left.\mathrm{S}_{\mathrm{B}}\right)$. We demonstrate that the ground state of different kinds of samples is determined by factors that one can affect such as temperature, preparation method, or intermolecular interactions. In this sense, knowledge of the exact electronic configuration of $\mathrm{MnPc}$ and of the fact that it can be modified opens up the possibility for manipulating the direction of the molecular magnetic anisotropy in molecular spintronics applications.

\section{ASSOCIATED CONTENT}

Comparison between the DOS of $\mathrm{S}_{\mathrm{A}}, \mathrm{S}_{\mathrm{B}}$, and $\mathrm{S}_{\mathrm{C}}$. The total densities of states are represented alongside all components (Mn 3d, C 2p, and N 2p). The gas-phase 100 $\mathrm{eV}$ photon energy experimental results in comparison with the calculated spectra of $\mathrm{S}_{\mathrm{A}}, \mathrm{S}_{\mathrm{B}}$, and metal-free phthalocyanine multiplied with the corresponding crosssection. 


\section{ACKNOWLEDGMENTS}

We acknowledge the Swedish Research Council (VR), the KAW foundation, and the Swedish National Infrastructure for Computing (SNIC). Support from eSSENCE and the ERC (ASD -project 247062) is also acknowledged.

\section{REFERENCES}

(1) Flood, A. H.; Stoddart, J. F.; Steuerman, D. W.; Heath, J. R. Whence Molecular Electronics. Science 2004, 306, 2055-2056.

(2) Bogani, L.; Wernsdorfer, W. Molecular Spintronics using Single-Molecule Magnets. Nat. Mater. 2008, 7, 179-186.

(3) Fu, Y. S.; Xue, Q. K.; Wiesendanger, R. Spin-Resolved Splitting of Kondo Resonances in the Presence of RKKY-Type Coupling. Phys. Rev. Lett. 2012, 108, 087203.

(4) Liu, L.; Yang, K.; Jiang, Y.; Song, B.; Xiao, W.; Li, L.; Zhou, H.; Wang, Y.; Du, S.; Ouyang, M.; et al. Reversible Single Spin Control of Individual Magnetic Molecule by Hydrogen Atom Adsorption. Sci. Rep. 2013, 3, 1210.

(5) Fu, Y. S.; Ji, S. H.; Chen, X.; Ma, X. C.; Wu, R.; Wang, C. C.; Duan, W. H.; Qiu, X. H.; Sun, B.; Zhang, P.; et al. Manipulating the Kondo Resonance through Quantum Size Effects. Phys. Rev. Lett. 2007, 99, 256601.

(6) Bauer, J.; Pascaul, J. I.; Franke, K. J. Microscopic Resolution of the Interplay of Kondo Screening and Superconducting Pairing: Mn-phthalocyanine Molecules Adsorbed on Superconducting Pb(111). Phys. Rev. B 2013, 87, 075125.

(7) Wende, H.; Bernien, M.; Luo, J.; Sorg, C.; Ponpandian, N.; Kurde, J.; Miguel, J.; Piantek, M.; Xu, X.; Eckhold, Ph.; et al. Substrate-induced Magnetic Ordering and Switching of Iron Porphyrin Molecules. Nat. Mater. 2007, 6, 516-520.

(8) Stróźecka,A.; Soriano,M.; Pascual,J.I.; Palacios,J.J. Reversible Change of the Spin State in a Manganese Phthalocyanine by Coordination of CO Molecule. Phys. Rev. Lett. $2012,109,147202$. 
(9) Heutz, S.; Mitra, C.; Wu, W.; Fisher, A. J.; Kerridge, A.; Stoneham, M.; Harker, T. H.; Gardener, J.; Tseng, H. H.; Jones, T. S.; et al. Molecular Thin Films: A New Type of Magnetic Switch. Adv. Mater. 2007, 19, 3618-3622.

(10) Lehmann, J.; Gaita-Ariño, A.; Coronado, E.; Loss, D. Quantum Computing with Molecular Spin Systems. J. Mater. Chem. 2009, 19, 1672-1677.

(11) Clemente-Juan, J. M.; Coronado, E.; Gaita-Ariño, A. Magnetic Polyoxometalates: from Molecular Magnetism to Molecular Spintronics and Quantum Computing. Chem. Soc. Rev. 2012, 41, 7464- 7478.

(12) Franke, K. J.; Schulze, G.; Pascual, J. I. Competition of Superconducting Phenomena and Kondo Screening at the Nanoscale. Science 2011, 332, 940-944.

(13) Goswami, T.; Misra, A. Ligand Effects toward the Modulation of Magnetic Anisotropy and Design of Magnetic Systems with Desired Anisotropy Characteristics. J. Phys. Chem. A 2012, 116, 5207-5215.

(14) Marom, N.; Kronik, L. Density Functional Theory of Transition Metal Phthalocyanines, II: Electronic Structure of MnPc and FePc - Symmetry and Symmetry Breaking. Appl. Phys. A: Mater. Sci. Process. 2009, 95, 165-172.

(15) Grobosch, M.; Mahns, B.; Loose, C.; Friedrich, R.; Schmidt, C.; Kortus, J.; Knupfer, M. Identification of the Electronic States of Manganese Phthalocyanine Close to the Fermi Level. Chem. Phys. Lett. 2011, 505, 122-125.

(16) Griffith, J. S.; Orgel, L. E. Ligand-Field Theory. Q. Rev. Chem. Soc. 1957, 11, 381-393.

(17) Petraki, F.; Peisert, H.; Hoffmann, P.; Uihlein, J.; Knupfer, M.; Chasse', T. Modification of the 3d-Electronic Configuration of Manganese Phthalocyanine at the Interface to Gold. J. Phys. Chem. C 2012, 116, 5121-5127.

(18) Williamson, B. E.; VanCott, T. C.; Boyle, M. E.; Misener, G. C.; Stillman, M. J.; Schatz, P. N. Determination of the Ground State of Manganese Phthalocyanine in an Argon Matrix Using Magnetic Circular Dichroism and Absorption Spectroscopy. J. Am. Chem. Soc. 1992, 114, 2412-2419.

(19) Kataoka, T.; Sakamoto, Y.; Yamazaki, Y.; Singh, V. R.; Fujimori, A.; Takeda, Y.; Ohkochi, T.; Fujimori, S.-I.; Okane, T.; Saitoh, Y.; et al. Electronic Configuration of Mn Ions in the $\pi$-d Molecular Ferromagnet $\beta$-Mn Phthalocyanine Studied by Soft X-ray 
Magnetic Circular Dichroism. Solid State Commun. 2012, 152, 806-809.

(20) Liao, M. S.; Watts, J. D.; Huang, M.-J. DFT Study of Unligated

Iand Ligated Manganese I Porphirins and Phthalocyanines. Inorg. Chem.

2005, 44, 1941-1949.(21) Wang, J.; Shi, Y.; Cao, J.; Wu, R. Magnetization and Magnetic

Anisotropy of Metallophthalocyanine Molecules from the First Principle Calculations. Appl. Phys. Lett. 2009, 94, 122502.

(22) Mitra, S.; Gregson, A. K.; Hatfield, W. E.; Weller, R. R. Single-Crystal Magnetic

Study on Ferromagnetic Manganese(II) Phthalocyanine. Inorg. Chem. 1983, 22, 1729-1732.

(23) Kroll, T.; Kraus, R.; Schönefelder, R.; Aristov, V.Yu.; Molodtsova, O. V. Transition Metal Phthalocyanines: Insight into the Electronic Structure from Soft X-ray Spectroscopy. J. Chem. Phys. 2012, 137, 054306.

(24) Blyth, R. R.; Delaunay, R.; Zitnik, M.; Krempasky, J.; Krempaska, R.; Slezak, J.; Prince, K. C.; Richter, R.; Vondracek, M.; Camilloni, R.; et al. The High Resolution Gas Phase Photoemission Beamline, Elettra. J. Electron Spectrosc. Relat. Phenom. 1999, 101-103, 959-964.

(25) Plekan, O.; Feyer, V.; Richter, R.; Coreno, M.; de Simone, M.; Prince, K. C.; Trofimov, A. B.; Gromov, E. V.; Zaytseva, I. L.; Schirmer, J. A Theoretical and Experimental Study of the Near Edge X-Ray Absorption Fine Structure (NEXAFS) and X-Ray Photoelectron Spectra (XPS) of Nucleobases: Thymine and Adenine. Chem. Phys. $2008,347,360-375$.

(26) Berkowitz, J. Photoelectron Spectroscopy of Phthalocyanine Vapors. J. Chem. Phys. $1979,70,2819-2828$.

(27) Kimura, K. Handbook of HeI Photoelectron Spectra of Fundamental Organic Molecules: Ionization Energies, Ab initio Assignments, and Valence Electronic Structure for 200 Molecules; Japan Scientific Societies Press/Halsted Press: New York, 1981.

(28) Frisch, M. J.; Trucks, G. W.; Schlegel, H. B.; Scuseria, G. E.; Robb, M. A.; Cheeseman, J. R.; Scalmani, G.; Barone, V.; Mennucci, B.; Petersson, G. A.; et al. Gaussian 09, revision C.01; Gaussian, Inc.: Wallingford, CT, 2009. 
(29) Becke, A. D. Density-Functional Thermochemistry. III. The Role of Exact Exchange. J. Chem. Phys. 1993, 98, 5648-5652.

(30) Rassolov, V. A.; Pople, J. A.; Ratner, M. A.; Windus, T. L. 6-31G* Basis Set for Atoms K through Zn. J. Chem. Phys. 1998, 109, 1223-1229.

(31) Balabanov, N. B.; Peterson, K. A. Systematically Convergent Basis Sets for Transition Metals. I. All-electron Correlation Consistent Basis Sets for the 3d Elements Sc-Zn. J. Chem. Phys. 2005, 123, 064107.

(32) Gorelsky, S. I.; Lever, A. B. P. Electronic Structure and Spectra of Ruthenium Diimine Complexes by Density Functional Theory and INDO/S. Comparison of the two Methods. J. Organomet. Chem. 2001, 635, 187-196.

(33) Brena, B.; Puglia, C.; de Simone, M.; Coreno, M.; Tarafder, K.; Feyer, V.; Banerjee, R.; Göthelid, E.; Sanyal, B.; Oppeneer, P. M.; et al. Valence-Band Electronic Structure of Iron Phthalocyanine: An Experimental and Theoretical Photoelectron Spectroscopy Study. J. Chem. Phys. 2011, 134, 074312.

(34) Green, J.; Decleva, P. Photoionization Cross-Sections: A Guide to Electronic Structure. Coord. Chem. Rev. 2005, 249, 209-228.

(35) Green, J. Variable Photon Energy Photoelectron Spectroscopy of Transition Metal Molecules. Acc. Chem. Res. 1994, 27, 131-137.

(36) Yeh, J. J. Atomic Calculation of Photoionization Cross-Sections and Asymmetry Parameters; Gordon and Breach Science Publishers: Langhorne, PA, 1993.

(37) Yeh, J. J.; Lindau, I. Atomic Subshell Photoionization Cross Sections and Asymmetry Parameters: $1 \leqslant Z \leqslant 103$. At. Data Nucl. Data Tables 1985, 32, 1-155.

(38) Nilson, K.; Åhlund, J.; Shariati, M.-N.; Schiessling, J.; Palmgren, P.; Brena, B.; Göthelid, E.; Hennies, F.; Huismans, Y.; Evangelista, F.; et al. Potassium-intercalated $\mathrm{H}_{2} \mathrm{Pc}$ Films: Alkali-induced Electronic and Geometrical Modifications. J. Chem. Phys. $2012,137,044708$. 


\section{FIGURES}

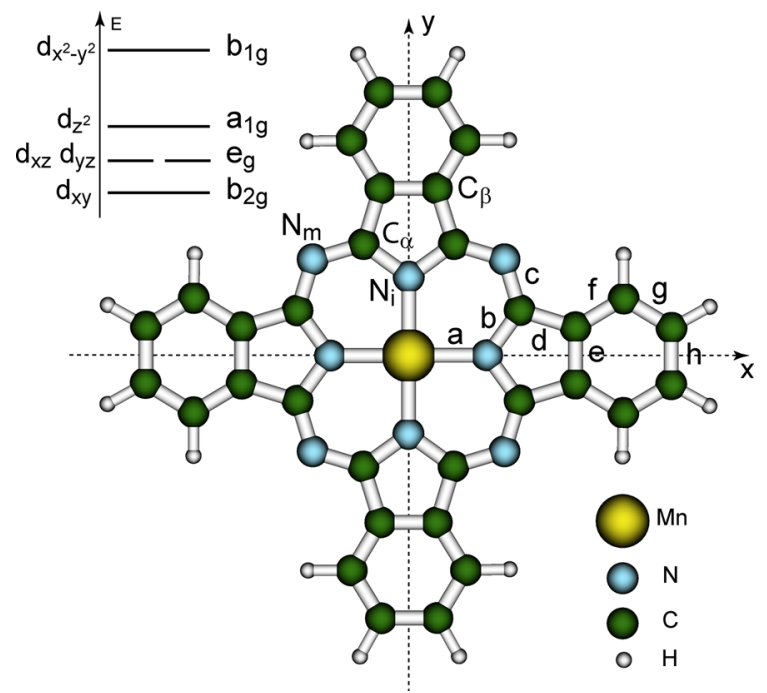

Figure 1. Schematic representation of the MnPc molecular geometry alongside a simplified and general view of the metal $3 \mathrm{~d}$ electronic levels according to ligand field theory (top left corner). The molecule is planar, and the $\mathrm{z}$ axis is perpendicular to the molecular plane.
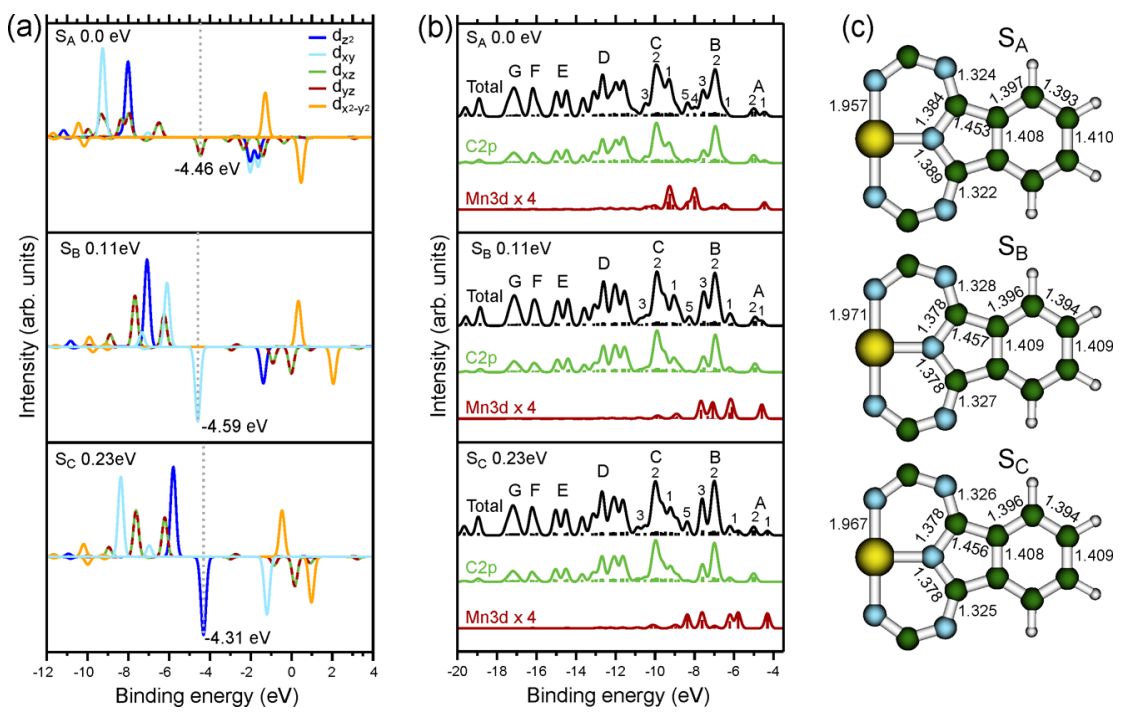

Figure 2. (a) Calculated PDOS for the Mn 3d levels of $S_{A}, S_{B}$, and $S_{C}$, respectively. The spectra were broadened using Gaussian functions of $0.3 \mathrm{eV}$ constant full width at halfmaximum (fwhm). The dashed lines represent the HOMO position. (b) Comparison between the TDOS, Mn 3d, and C 2p PDOS of $S_{A}, S_{B}$, and $S_{C}$, respectively. Both bar 
graphs and broadened spectra $(0.3 \mathrm{eV}$ constant fwhm) are shown. The Mn $3 \mathrm{~d}$ intensities were multiplied by a factor of four for clarity. (c) Segment of MnPc with the relevant bond lengths for each structure.

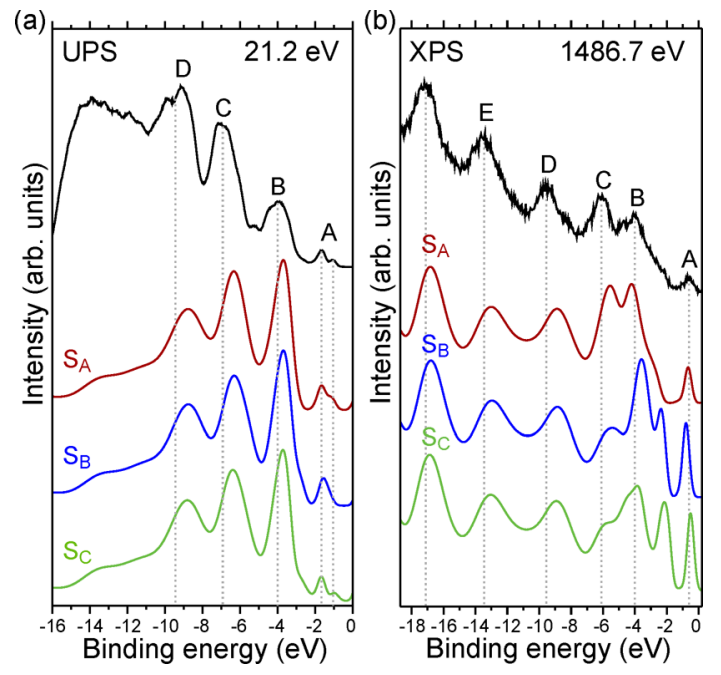

Figure 3. Comparison between the thin-film experimental results (UPS and XPS) and the computed spectra at (a) 21.2 and (b) $1486.7 \mathrm{eV}$ photon energies. The calculated DOS were broadened and shifted as described in the Computational Methods section.
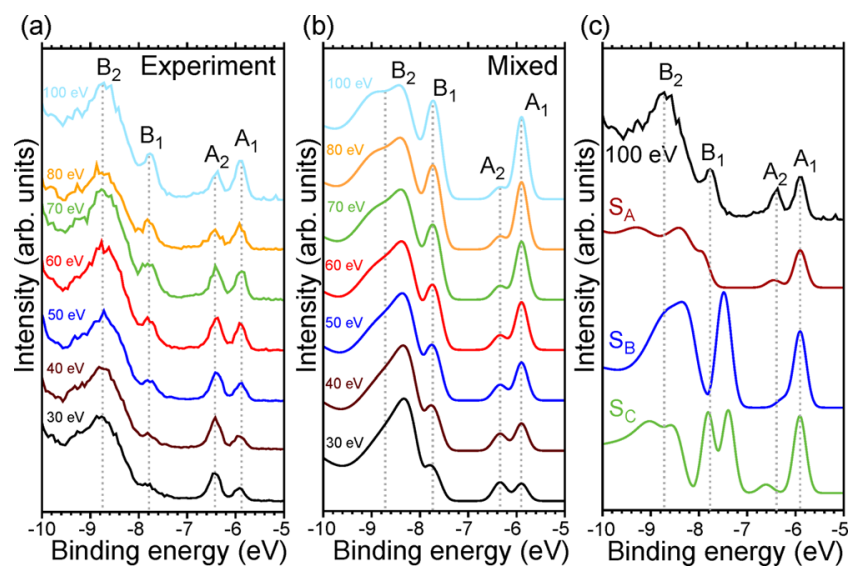

Figure 4. Comparison between (a) the gas-phase experimental results and (b) the simulated mixed electronic configuration spectra for seven different photon energies (30-100 eV). The dashed lines mark the positions of the calculated bands. (c) Comparison between the $100 \mathrm{eV}$ calculated results and the gas-phase experiment performed at the same photon energy. The dashed lines mark the positions of the experimental peaks. All calculated spectra were broadened, normalized, and shifted as 
described in the Computational Methods section. 


\section{TABLES}

Table 1. Calculated Electronic Properties and Electronic Configurations of the S, $\mathrm{S}$ and $\mathrm{S}^{\mathrm{a})}$

\begin{tabular}{|c|c|c|c|c|}
\hline structure & occupation & HOMO symm & orbital type & $\begin{array}{c}\text { HOMO-LUMO } \\
\text { gap }\end{array}$ \\
\hline $\mathrm{S}_{\mathrm{A}}$ & $\mathrm{b}_{2 \mathrm{~g}}{ }^{1} \mathrm{e}_{\mathrm{g}}{ }^{3} \mathrm{a}_{1 \mathrm{~g}}{ }^{1} \mathrm{~b}_{2 \mathrm{~g}}{ }^{0}$ & $\mathrm{e}_{\mathrm{g}}$ & $\mathrm{d}_{\mathrm{xz}}, \mathrm{d}_{\mathrm{yz}}$ & $1.35 \mathrm{eV}$ \\
\hline $\mathrm{S}_{\mathrm{B}}$ & $\mathrm{b}_{2 \mathrm{~g}}{ }^{2} \mathrm{e}_{\mathrm{g}}{ }^{2} \mathrm{a}_{1 \mathrm{~g}}{ }^{1} \mathrm{~b}_{2 \mathrm{~g}}{ }^{0}$ & $\mathrm{~b}_{2 \mathrm{~g}}$ & $\mathrm{~d}_{\mathrm{xy}}$ & $1.65 \mathrm{eV}$ \\
\hline $\mathrm{S}_{\mathrm{C}}$ & $\mathrm{b}_{2 \mathrm{~g}}{ }^{1} \mathrm{e}_{\mathrm{g}}{ }^{2} \mathrm{a}_{1 \mathrm{~g}}{ }^{2} \mathrm{~b}_{2 \mathrm{~g}}{ }^{0}$ & $\mathrm{a}_{1 \mathrm{~g}}$ & $\mathrm{~d}_{\mathrm{z}}{ }^{-2}$ & $1.32 \mathrm{eV}$ \\
\hline
\end{tabular}

a) To describe the occupancy of the different configuration, we have listed the molecular orbitals in the same order. The actual energy ordering is represented in Figure 2a. 